\title{
The Abelian Groups of Large Order: Perspective from (Fuzzy) Subgroups of Finite p-Groups
}

\author{
Sunday Adesina Adebisi ${ }^{1, ~ *}$, Mike Ogiugo ${ }^{2}$, Michael Enioluwafe ${ }^{3}$ \\ ${ }^{1}$ Department of Mathematics, Faculty of Science, University of Lagos, Lagos, Nigeria \\ ${ }^{2}$ Department of Mathematics, School of Science, Yaba College of Technology, Lagos, Nigeria \\ ${ }^{3}$ Department of Mathematics, Faculty of Science, University of Ibadan, Ibadan, Nigeria
}

Email address:

adesinasunday@yahoo.com (S. A. Adebisi), ekpenogiugo@gmail.com (M. Ogiugo), michael.enioluwafe@gmail.com (M. Enioluwafe)

${ }^{*}$ Corresponding author

\section{To cite this article:}

Sunday Adesina Adebisi, Mike Ogiugo, Michael Enioluwafe. The Abelian Groups of Large Order: Perspective from (Fuzzy) Subgroups of Finite p-Groups. Mathematics and Computer Science. Vol. 6, No. 3, 2021, pp. 45-48. doi: 10.11648/j.mcs.20210603.11

Received: MM DD, 2021; Accepted: MM DD, 2021; Published: MM DD, 2021

\begin{abstract}
In the recent past, results have shown that Nilpotent groups such as p-groups, have normal series of finite length. Any finite p-group has many normal subgroups and consequently, the phenomenon of large number of non-isomorphic subgroups of a given order. This makes it an ideal object for combinatorial and cohomological investigations. Cartesian product (otherwise known as the product set) plays vital roles in the course of synthesizing the abstract groups. Previous studies have determined the number of distinct fuzzy subgroups of various finite p-groups including those of square-free order. However, not much work has been done on the fuzzy subgroup classification for the nilpotent groups formed from the Cartesian products of p-groups through their computations. Here, part of our intention is therefore trying to make some designs so as to classify the nilpotent groups formed from the Cartesian products of p-groups through their computations. The Cartesian products of p-groups were taken to obtain nilpotent groups. Results up to two dimensions are now obtainable. In this paper, the fuzzy subgroups of the nilpotent product of two abelian subgroups of orders $2^{n}$ and 128 . The integers $n \geq 7$ have been successfully considered and the derivation for the explicit formulae for its number distinct fuzzy subgroups were calculated. Some methods were once being used in counting the chains of fuzzy subgroups of an arbitrary finite $p$-group $G$. Here, the adoption of the famous Inclusion-Exclusion principle is very necessary and imperative so as to obtain a reasonable, and as much as possible accurate.
\end{abstract}

Keywords: Finite $p$-Groups, Abelian Group, Fuzzy Subsets, Fuzzy Subgroups, Inclusion-Exclusion Principle, Maximal Subgroups, Nilpotent Group

\section{Introduction}

Many methods, techniques and approaches have been used for the classification of which some are obtainable (see [6] and [10]). In this work, one of the essential role in solving counting problems is played by adopting the Inclusion-Exclusion Principle. The process leads to some recurrence relations from which the solutions are finally computed with ease. In the process of our computation, the use of GAP (Group Algorithm and Programming) was actually applied. In the actual sense, some elements of fuzzy logics come to play in the process of computing certain numbers of subgroups. And so representation, management, manipulation and processing of data and information are very imperative in this fast growing, developing and advancing world, most especially, the nonstatistical aspect and the uncertainties. As a result, course in 1965. Notwithstanding, the story of fuzzy logic started much more earlier, it was specially designed, mathematically to represent uncertainty and vagueness. It also provides formalized tools used for the imprecision intrinsic to many problems. In the recent few years, the problem of classifying the fuzzy subgroups of a finite group has experienced a very rapid and dynamic developments. One particular case or the other have been treated by several papers such as the finite abelian as well as the non-abelian groups. In (Akgual, 1988) (see [13]), the number of fuzzy subgroups of a finite cyclic group of square-free order which are distinct has been 
determined, while (Bhattachachanya, 1997), (Blackburn, 1960) [14], (Blackburn, 1965 (see [16])) and (Gagola, 1999) (see [15]) deal with this number for cyclic groups of order $\mathrm{p}^{\mathrm{n}} \mathrm{q}^{\mathrm{m}}$, where $\mathrm{p}$ and $\mathrm{q}$ are prime numbers. Moreover, in (Gegang, 2005), a recurrence relation is indicated which can successfully be used to count the number of distinct fuzzy subgroups for two classes of finite abelian groups, namely: the arbitrary finite cyclic groups and finite elementary abelian p- groups. The explicit formula obtained gave rise to an expression of a wellknown central Delannoy numbers (Cossey, 1982). Sequel to the discovery of the existence of p-groups, various work have been done while researches are continuously being carried out from day to day by a number of eminent personalities. Joseph L. Lagrange, in 1771 had a theorem accredited to him based on finite Group. Meanwhile, he did not prove this theorems all he did, essentially, was to discuss some special cases. The first complete proof of the theorem was provided by Abbati. In 1872, the Normegran mathematician L. Sylow had a collection of theorems on finite group named after him. Moreover, the Sylow theorems have been proved in a number of different ways, and the history of the proofs themselves are the subjects of many papers. Wielandt in 1959 used combinatorial arguments to show part of the Sylow theorems. Frattini had his argument on Sylow subgroups of a normal subgroup which was slightly generalized by Burnside as Burnside's fusion theorem. Others are Brauer, Gorenstein and J. L. Alperin. Some of the current and dynamic researches are being carried on some categories of p-groups by Y. Berkovich and Zvonimir Janko who are from Israel and Germany respectively.

\section{The Historical Backgrounds of Logical Fuzziness}

The laws of Thought" as could be called was posited by Aristotle to devise concise theory of logic, and later mathematics. One of these, the law of the Excluded Middle", states that every proposition must either be True $(\mathrm{T})$ or $\mathrm{t}(\mathrm{F})$. Parminedes had first proposed the first version of this law (by 400B.C.) but there were strong and immediate objections part of this was thefact that things could be simultaneously True and also not True. This was, in particular proposed by Heraclitus. It was Plato who laid the foundation for what would become Fuzzy logic, indicating that there was a third region (beyond $\mathrm{T}$ and $\mathrm{F}$ ) where these opposites Itumbled about". A systematic alternative to the bi-valued logic of Aristotle was first proposed by Lukasiewicz around 1920. Then, he described a three-valued logic, along with the mathematics to accompany it. The third value he proposed can best be translated as the term possible" and he assigned it a NUMERIC VALUE between $\mathrm{T}$ and F. He eventually proposed an entire notation and axiomatic system from which he hoped to derive modern mathematics. It was later that he explored four-valued logics, five-valued logics, and then declared that in principle, there was nothing to prevent the derivation of an infinite-valued logic. Lukasiewicz felt that three- and infinitevalued logics were the most intriguing, but he ultimately settled on a four-valued logic because it seemed to be the most easily adaptable to the Aristotelian logic. Similar to Lukasiewicz's idea was a proposition of a three-valued logic by Knuth. From this, he speculated that mathematics would become even more elegant than in traditional bi-valued logic.

\section{Modern Theory and Techniques of Logical Arithmetic}

In his seminal work on Fuzzy Sets", the notion of an infinite-valued logic was introduced by Zadeh. He described the mathematics of Fuzzy set theory, this he extended to Fuzzy logic. The theory thus proposed allows the membership function (or the values $\mathrm{T}$ and $\mathrm{F}$ ) to operate over the range of real numbers $[0 ; 1]$. New operations for calculus of logic were proposed and showed to be in principle at least a generalization of classic logic. Fuzzy logic provides an inference morphology that enables approximate human reasoning capabilities to be applied to knowledge-based systems. The theory of Fuzzy logic provides a mathematical strength to capture the uncertainties associated with human cognitive processes, such as thinking and reasoning. Meanwhile, it is evident that our world is such an uncertain one, which is full of various uncertainties and mysterious incidences. The executions of imprecise knowledge to arrive at definite decisions was necessitated by the fact that human beings barely comprehend quantitatively some decisionmaking and problem-solving tasks that are somehow very complex one way or the other. This culminated in the inception and advent of fuzzy set theory which exploits approximate data and uncertainty to generate decisions. Date back to Plato and Lukaievic in the 1900s at some stage known as the Many-valued logic, Zadeh formalized fuzzy logic in the 1960s. Fuzzy groups was developed by Rosenfield in 1971. P.S. Das was the brain behind level subgroups. He used this for the characterization of the fuzzy subgroups of finite groups. The notion of equivalence of fuzzy subgroups was introduced by Morali and Makamba. From here, various other advances have emanated through a host of researhers such as Volf, Branimir and Tepavcevic, Degang, Bentea, Ghafur and Sulaiman, Ajmal, Morden, Dixit, Laszlo, Zhang and Zou. Others are Jain, Mashinchi, Mukaidonon, Iranmaresh, Maraghi, Bhattacharya and Mukherjee, to mention but a few. Among the most recent contributors include: Marius Tarnauceanu. He has worked in collaboration with the host of eminent researchers such as Bentea and others. His work so far has been able to give some relevant clue on the classification of fuzzy subgroups for a class of finite p-groups including some abelian, elementary abelian as well as the so called non-abelian types. Representation, management, manipulation and processing of data and information are very imperative in this fast growing, developing and advancing world, most especially the nonstatistical and uncertainties. Hence, Fuzzy sets were introduced by Zadeh in 1965. Even though, the story of Fuzzy logic started much more earlier, it was specially 
designed mathematically to represent uncertainty and vagueness. It was also, to provide formalized tools for dealing with the imprecision intrinsic to many problems. The conventional approaches to knowledge representation lack the means for representing the meaning of Fuzzy concepts. As a consequence, the approaches based on first order logic and classical probability theory do not provide an appropriate conceptual framework for dealing with the representation of commonsense knowledge, since such knowledge is by its nature both lexically imprecise and non-categorical. In a large measure, the development of Fuzzy logic was motivated by the need for a conceptual framework that can address the issue of uncertainty and lexical imprecision.

\section{Fuzzy Sets}

The idea of a fuzzy set was derived from the generalization of the concept of a crisp set. Unlike in classical set theory where membership of an element of a set is viewed in binary terms of a bivalent nature (is a member of or is not a member of), the generalization of classical sets to fuzzy sets allows for elements of a set to partially belong to that set.

\section{Basic Definitions and Terms}

Suppose that $(G, e)$ is a group with identity $e$. Let $S(G)$ denote the collection of all fuzzy subsets of $G$. An element $\lambda$ $\in S(G)$ is said to be a fuzzy subgroup of $G$ if the following two conditions are satisfied

$$
\begin{gathered}
\lambda(\mathrm{ab}) \geq \min \{\lambda(\mathrm{a}), \lambda(\mathrm{b})\}, \forall \mathrm{a}, \mathrm{b} \in \mathrm{G} ; \\
\text { (ii) } \lambda\left(\mathrm{a}^{-1} \geq \lambda \text { (a) for any } \mathrm{a} \in \mathrm{G} .\right.
\end{gathered}
$$

And, since $\left(a^{-1}\right)^{-1}=a$, we have that $\lambda\left(a^{-1}\right)=\lambda(a)$, for any $a$ $\in G$.

Also, by this notation and definition, $\lambda(e)=\sup \lambda(G)$. [Marius [7]]. Define by $M_{1}, M_{2}, \ldots, M_{t}$, the maximal subgroups of $G$, and denote by $h(G)$ the number of chains of subgroups of $G$ which ends in $G$.

Theorem [Marius [7]: The set $F L(G)$ possessing all fuzzy subgroups of $G$ forms a lattice under the usual ordering of fuzzy set inclusion. This is called the fuzzy subgroup lattice of $G$.

We define the level subset: $\lambda G_{\beta}=\{a \in G / \lambda(a) \geq \beta\}$ for each $\beta \in[0,1]$ The fuzzy subgroups of a finite $p$-group $G$ are thus, characterized, based on these subsets. In the sequel, $\lambda$ is a fuzzy subgroup of $G$ if and only if its level subsets are subgroups in $G$. This theorem gives a link between $F L(G)$ and $L(G)$, the classical subgroup lattice of $G$.

Moreover, some natural relations on $S(G)$ can also be used in the process of classifying the fuzzy subgroups of a finite $q$ group $G$ (see [9] and [10]). One of them is defined by: $\lambda \sim \gamma$ iff $(\lambda(a)>\lambda(b) \Leftarrow \Rightarrow v(a)>v(b), \forall a, b \in G)$. Also, two fuzzy subgroups $\lambda, \gamma$ of $G$ are said to be distinct if $\lambda \nsim v$.

As a result of this development, let $G$ be a finite $p$-group and suppose that $\lambda: G \rightarrow[0,1]$ is a fuzzy subgroup of $G$. Put $\lambda(G)=\left\{\beta_{1}, \beta_{2}, \ldots, \beta_{k}\right\}$ with the assumption that $\left.\beta_{1}<\beta_{2}>\cdots\right\rangle$ $\beta_{k}$. Then, ends in $G$ is determined by $\lambda$.

$$
\lambda G_{\beta 1} \subset \lambda G_{\beta 2} \subset \cdots \subset \lambda G_{\beta k}=G
$$

Also, we have that:

$$
\lambda(a)=\beta_{t} \Leftarrow \Rightarrow t=\max \left\{r / a \in \lambda G_{\beta r}\right\} \Leftrightarrow \Rightarrow \in \lambda G_{\beta t} \downarrow \lambda G_{\beta t-1},
$$
for any $a \in G$ and $t=1, \ldots, k$, where by convention, set $\lambda G_{\beta 0}$ $=\emptyset$.

\section{The Techniques}

The usable method that will be used or applied in counting the chains of fuzzy subgroups of an arbitrary finite $p$-group $G$ is described. For cases of some simple and moderate order. (see $[1,2]$ and [3]). Suppose that $M_{1}, M_{2}, \ldots, M_{t}$ are the maximal subgroups of $G$, and denote by $h(G)$ the number of chains of subgroups of $G$ which ends in $G$. By simply applying the technique of computing $h(G)$, using the application of the Inclusion-Exclusion Principle, we have that:

$$
h(G)=2\left(\sum_{r=1}^{t} h\left(M_{r}\right)-\sum_{1 \leq r_{1}<r_{2} \leq t} h\left(M_{r_{1}} \cap M_{r_{2}}\right)+\cdots+(-1)^{t-1} h\left(\bigcap_{r=1}^{t} M_{r}\right)\right)(\#)
$$

In [8], (\#)(2) was used to obtain the formulas for some positive integers $n$.

Theorem (*) [Marius][10]: The number of distinct fuzzy subgroups of a finite $p$-group of order $p^{n}$ which have a cyclic maximal subgroup is:

$$
\begin{gathered}
\mathrm{h}\left(\mathbb{Z}_{p^{n}}\right)=2^{\mathrm{n}} \\
\mathrm{h}\left(\mathbb{Z}_{p} \times \mathbb{Z}_{p^{n-1}}\right)=\mathrm{h}\left(\mathrm{M}_{\mathrm{p}} \mathrm{n}\right)=2^{\mathrm{n}-1}(2+(\mathrm{n}-1) \mathrm{p})
\end{gathered}
$$

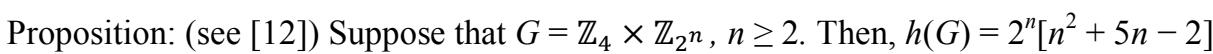

Proof: $G$ has three maximal subgroups of which two are isomorphic to $\mathbb{Z}_{2} \times \mathbb{Z}_{2^{n}}$ and the third is isomorphic to $\mathbb{Z}_{4} \times \mathbb{Z}_{2^{n-1}}$. Hence

$$
\begin{gathered}
\mathrm{h}\left(\mathbb{Z}_{4} \times \mathbb{Z}_{2^{n}}\right)=2 \mathrm{~h}\left(\mathbb{Z}_{2} \times \mathbb{Z}_{2^{n}}\right)+2^{1} \mathrm{~h}\left(\mathbb{Z}_{2} \times \mathbb{Z}_{2^{n-1}}\right)+2^{1} \mathrm{~h}\left(\mathbb{Z}_{2} \times \mathbb{Z}_{2^{n-2}}\right) \\
+2^{3} \mathrm{~h}\left(\mathbb{Z}_{2} \times \mathbb{Z}_{2^{n-3}}\right)+2^{4} \mathrm{~h}\left(\mathbb{Z}_{2} \times \mathbb{Z}_{2^{n-4}}\right)+\cdots+2^{\mathrm{n}-2} \mathrm{~h}\left(\mathbb{Z}_{2} \times \mathbb{Z}_{2^{2}}\right)
\end{gathered}
$$

We have that: $h\left(\mathbb{Z}_{4} \times \mathbb{Z}_{2^{n-1}}\right)=2^{n-1}\left[(n-1)^{2}+5(n-1)-2\right]=2^{n-1}\left[n^{2}+3 n-6\right], n>2$ 
proposition A (see [4]): Suppose that $\mathrm{G}=\mathbb{Z}_{16} \times \mathbb{Z}_{2^{n}}$

Then $\mathrm{h}(\mathrm{G})=\frac{1}{3}\left(2^{n+2}\right)\left(n^{3}+12 n^{2}+17 \mathrm{n}-24\right)+2^{n}(200)+\frac{1}{3}\left(2^{n+1}\right) \sum_{k=1}^{n-5}\left[\left((n-k)^{3}+12(n-k)^{2}+17(\mathrm{n}-\mathrm{k})-24\right)\right.$

Proposition B (see [5 and 11]): Suppose that $G=\mathbb{Z}_{32} \times \mathbb{Z}_{2^{n}}$

$$
\text { Then } h(G)=2\left[h\left(\mathbb{Z}_{16} \times \mathbb{Z}_{2^{n}}\right)+h\left(\mathbb{Z}_{16} \times \mathbb{Z}_{2^{n-1}}\right)\right]+\sum_{k=1}^{n-6} 2^{k+1}\left(h\left(\mathbb{Z}_{16} \times \mathbb{Z}_{2^{n-1-k}}\right)\right)
$$

Computation for $\mathrm{G}=\mathbb{Z}_{\mathbf{1 2 8}} \times \mathbb{Z}_{\mathbf{2}^{\mathbf{n}}}$

Suppose that $G=\mathbb{Z}_{64} \times \mathbb{Z}_{64}$ Then,

$$
\begin{gathered}
\mathrm{h}(\mathrm{G})=2 \mathrm{~h}\left(\mathbb{Z}_{32} \times \mathbb{Z}_{2^{6}}\right) \\
\text { If } \mathrm{G}=\mathbb{Z}_{128} \times \mathbb{Z}_{2^{7}}, \text { then, } \mathrm{h}(\mathrm{G})=2\left[\mathrm{~h}\left(\mathbb{Z}_{128} \times \mathbb{Z}_{2^{6}}\right)+\mathrm{h}\left(\mathbb{Z}_{64} \times \mathbb{Z}_{2^{7}}\right)-\mathrm{h}\left(\mathbb{Z}_{64} \times \mathbb{Z}_{2^{6}}\right)\right. \\
\text { Now, let } \mathrm{G}=\mathbb{Z}_{64} \times \mathbb{Z}_{2^{n}, \text { then }} \\
\mathrm{h}(\mathrm{G})=2 \mathrm{~h}\left(\mathbb{Z}_{64} \times \mathbb{Z}_{2^{n}}\right)-2 \mathrm{~h}\left(\mathbb{Z}_{64} \times \mathbb{Z}_{2^{n-1}}\right)+2 \mathrm{~h}\left(\mathbb{Z}_{128} \times \mathbb{Z}_{2^{n-1}}\right) \\
=2 \mathrm{~h}\left(\mathbb{Z}_{64} \times \mathbb{Z}_{2^{n}}\right)+2 \mathrm{~h}\left(\mathbb{Z}_{64} \times \mathbb{Z}_{2^{n-1}}\right)+4 \sum_{k=1}^{t-2} 2^{k-1}\left(h\left(\mathbb{Z}_{64} \times \mathbb{Z}_{2^{n-1-k}}\right)\right)-2^{t} \mathrm{~h}\left(\mathbb{Z}_{64} \times \mathbb{Z}_{2^{n-t}}\right)+2^{t} \mathrm{~h}\left(\mathbb{Z}_{128} \times \mathbb{Z}_{2^{n-t}}\right)(4)
\end{gathered}
$$

where, $\mathrm{n}-\mathrm{t}=6$, implying that $\mathrm{t}=\mathrm{n}-6$

Therefore

$$
\mathrm{h}(\mathrm{G})=2 \mathrm{~h}\left(\mathbb{Z}_{64} \times \mathbb{Z}_{2^{n}}\right)+2 \mathrm{~h}\left(\mathbb{Z}_{64} \times \mathbb{Z}_{2^{n-1}}\right)+4 \sum_{k=1}^{n-8} 2^{k-1}\left(h\left(\mathbb{Z}_{64} \times \mathbb{Z}_{2^{n-1-k}}\right)\right)-2^{\mathrm{n}-6} \mathrm{~h}\left(\mathbb{Z}_{64} \times \mathbb{Z}_{2^{6}}\right)+2^{\mathrm{n}-6} \mathrm{~h}\left(\mathbb{Z}_{128} \times \mathbb{Z}_{2^{6}}\right)
$$

\section{Conclusion}

For certain finite subgroups of somewhat large order, the number of distinct fuzzy subgroups abelian subgroups have been computed thus. Hence, this forms some necessary basis over which the classification of finite nilpotent groups which are of course p-groups are generally classified.

\section{References}

[1] Adebisi S. A., M. Ogiugo and M. EniOluwafe (2020) Computing the Number of Distinct Fuzzy Subgroups for the Nilpotent p-Group of International J. Math. Combin. Vol. 1 (2020), 86-89.

[2] Adebisi S. A., M. Ogiugo and M. EniOluwafe (2020) Determining The Number of Distinct Fuzzysubgroups For The Abelian Structure: Transactions of the Nigerian Association of Mathematical Physics Volume 11, (January - June, 2020 Issue), pp 5-6.

[3] Adebisi S. A., M. Ogiugo and M. EniOluwafe (2020) The Fuzzy Subgroups For The Abelian Structure: Z8 $\times Z 2 n ; n>2$. Journal of the Nigerian Mathematical Society, Vol. 39, Issue 2, pp. 167-171.

[4] Adebisi S. A., M. Ogiugo and M. EniOluwafe Fuzzy Subgroups For (The Cartesian Product of) The Abelian Structure Journal of The Nigerian Mathematical Society (Submitted for publication).

[5] Adebisi S. A., M. Ogiugo and M. EniOluwafe Fuzzy Subgroups For the Abelian Structurigerian Journal of Mathematics and Applications (Submitted).

[6] Mashinchi M., Mukaidono M. (1992). A classification of fuzzy subgroups. Ninth Fuzzy System Symposium, Sapporo,
Japan, 649-652.

[7] Tarnauceanu Marius. (2013). Classifying fuzzy subgroups for a class of finite $p$-groups. "ALL CUZa" Univ. Iasi, Romania pp 30-39.

[8] Tarnauceanu, Marius. (2012). Classifying fuzzy subgroups of finite nonabelian groups. Iran. J. Fussy Systems. (9) 33-43.

[9] Tarnauceanu, M., Bentea, L.(2008). A note on the number of fuzzy subgroups of finite groups, Sci. An. Univ.”ALL. Cuza" Iasi, Matt., (54) 209-220.

[10] Tarnauceanu, Marius., Bentea, L. (2008). On the number of fuzzy subgroups of finite abelian groups, Fuzzy Sets and Systems (159), 10841096, doi: 10.1016/j.fss.2017.11.014.

[11] S. A. ADEBISI, M. Ogiugo and M. EniOluwafe (2020) NEW DISCOVERIES ON THE FINITE $p$-GROUPS OF ORDER: Transnational Journal of Mathematical Analysis and Applications Vol. 8, Issue 1, 2020, Pages 17-23 ISSN 23479086 Published Online on December 21, Jyoti Academic Press http://jyotiacademicpress.org.

[12] S. A. ADEBISI, M. Ogiugo and M. EniOluwafe (2020) DETERMINING THE NUMBER OF DISTINCT FUZZYSUBGROUPS FOR THE ABELIAN STRUCTURE: Transactions of the Nigerian Association of Mathematical Physics Volume 11, (January - June, 2020 Issue), pp 5-6.

[13] Akgual, M.(1988). Some Properties of Fuzzy Subgroups. J. Maths. Anal. Appl. 133, 93-100.

[14] Blackburn, N. (1960). Nilpotent groups in which the derived group has two generators. J. Lond. Math. Soc., 35, 33-35.

[15] Gagola, S. M. and Lewis, M. L. (1999). A Character theoretic condition characterizing nilpotent groups, Comm. Algebra 27 (3), 1053-1056.

[16] Blackburn, N. (1965). Conjugacy in nilpotent groups. Proc. Amer. Math. Soc. 16, 143-148. 